the last six months, presence of decayed teeth, filled teeth, and missing teeth. Binary and multinomial logistic regression analyses were conducted weighted for complex sample design. Results No association between colour/race and time since last dental visit on the fully fitted model was observed. For the comparison between visits due to preventive/check-up and pain or extraction, the association remained significant only for Pardos compared to Whites after the adjustments (RRR 1.63 ; 95\% CI 1.11-2.38). In contrast, there was no association between colour/race and visiting the dentist due to treatment. Results from the adjusted model showed statistical differences only for Blacks, and this group was 1.48 times (95\% CI 1.10-1.97) more likely to visit the public dental service compared to Whites.

Conclusion Racial inequalities in dental service utilisation were evident for middle-aged adults in Brazil. For some outcomes, the study showed that colour/race inequalities were not fully explained by the covariates investigated. Further multilevel analysis will be conducted to better understand these inequalities.

\section{P30 DOES THE QUALITY OF SOCIAL RELATIONSHIPS BUFFER THE ASSOCIATION OF SOCIAL DISADVANTAGE WITH ALLOSTATIC LOAD? AN ANALYSIS OF ADULTS FROM THE UK HOUSEHOLD LONGITUDINAL STUDY}

${ }^{1} \mathrm{P}$ Rouxel ${ }^{*},{ }^{2} \mathrm{~T}$ Chandola, ${ }^{3} \mathrm{M}$ Benzeval. ${ }^{1}$ Social Science, UCL Institute of Education, London, UK; ${ }^{2}$ Institute for Social and Economic Research, University of Essex, Colchester, UKi ${ }^{3} \mathrm{CMIST}$ and Social Statistics, University of Manchester, Manchester, UK

\subsection{6/jech-2017-SSMAbstracts.132}

Background Positive support from social relationships is associated with better health, although it remains debatable whether positive social support buffers against the negative effects of social disadvantage on health. Moreover, few studies have considered both positive and negative relationship features from different networks (partner, relatives, and friends) and their association with allostatic load, a multisystem physiological dysregulation index, and none have examined whether the association of social disadvantage with higher levels of allostatic load reduces among those with more positive social support (the stress buffering role of positive social support).

Methods This study examined data from 7928 adults from the UK Household Longitudinal Study (wave 2-2010/11). Positive and negative social relationships were assessed within networks (partner, relatives, friends). Allostatic load (range 0-10) was measured by summing eleven risk scores across neuroendocrine, immune, metabolic, cardiovascular and anthropometric systems. We used negative binomial regression models to examine the association of positive social support and negative social interactions with allostatic load, controlling for socioeconomic/demographic, health, behavioural and personality factors.

Results Positive support from all networks was associated with lower allostatic load. Similarly, negative interactions from all networks were associated with higher allostatic load. However, after adjusting for all covariates, only positive support from partners and relatives, and negative interactions with friends were associated with allostatic load. Men with degree qualifications and supportive partners had lower allostatic load (2.3; 95\% CI: 2.2-2.5) than highly educated men with low partner support $(2.7$; 95\% CI: 2.4-3.0); positive support did not buffer the association of low education with allostatic load.
Unemployed adults with supportive partners had lower allostatic load $(2.4 ; 95 \% \mathrm{CI}: 1.9-2.8)$ compared to unemployed adults with low partner support (3.2; 95\% CI: 2.4-4.0).

Conclusion Positive social support from partners appears to buffer the effect of unemployment on allostatic load. However, contrary to the stress buffering hypothesis, highly educated men appear to benefit more from supportive partners than men and women with no qualifications.

\section{P31 ACCESS TO PRIMARY CARE FOR SOCIO-ECONOMICALLY DISADVANTAGED OLDER PEOPLE IN RURAL AREAS}

${ }^{1}$ JA Ford*, ${ }^{1}$ AP Jones, ${ }^{2} \mathrm{G}$ Wong, ${ }^{1} \mathrm{~A}$ Clark, ${ }^{1} \mathrm{~T}$ Porter, ${ }^{1} \mathrm{~N}$ Steel. ${ }^{1}$ Norwich Medical School, University of East Anglia, Norwich, UK; ${ }^{2}$ Nuffield Department of Primary Care, University of Oxford, Oxford, UK

\subsection{6/jech-2017-SSMAbstracts.133}

Background Our previous realist review and qualitative research found that socio-economically disadvantaged older people in rural areas face personal, community and health care barriers to obtaining an appointment in primary care. We described these barriers with context-mechanism-outcome (CMO) configurations. Example contexts were lifelong poverty, education, previous healthcare experience, transport, mechanisms were health literacy, assertiveness and convenience, and the outcome was obtaining an appointment. We aimed to explore these CMO configurations using Structural Equation Modelling (SEM) in a national linked dataset.

Methods Individual patient data from Wave 6 of the English Longitudinal Study of Ageing (ELSA) was linked with practice data from the GP Patient Survey (GPPS). Participants in the lowest socio-economic group and living in a rural area were included. Variables from ELSA and GPPS were available for nine complete CMOs. Confirmatory factor analysis was used to generate seven latent variables for unobserved concepts such as lifelong poverty and previous healthcare experience. CMOs were analysed in one overall SEM with multiple mediation paths; contexts were treated as the exposure, mechanisms as the mediator, and the common outcome was ability to obtain an appointment. Stata and MPlus was used to estimate standardised coefficients and 95\% confidence intervals using robust maximum likelihood.

Results 276 patients from 178 different GP surgeries were included. We found statistically significant direct or indirect effects for two of the nine CMOs. The ease of getting through to the surgery was statistically significantly associated with being able to get an appointment ( $\beta$ 0.52, 0.42 to 0.61$)$ with an indirect, statistically significantly, mediated effect through convenience ( $\beta 0.14,0.07$ to 0.21 ). Health care experience was not directly associated with getting an appointment $(\beta-0.04,-0.19$ to 0.12$)$, but a statistically significant indirect effect through convenience existed ( $\beta$ 0.10, 0.04 to 0.16 ). Model fit showed mixed results (RMSEA 0.05, CFI 0.923, TLI 0.901) Analysis was limited because there were not data for all theoretical concepts. Furthermore, continuous variables were only included to obtain adequate model fit.

Conclusion We found that obtaining an appointment was both directly associated with the ease of the booking system, and mediated through the mechanism of perceived convenience. We also found a mediated effect from previous health care experience to obtaining an appointment through perceived convenience. 